\title{
Hyper-excitabilité bronchique non allergénique
}

L'hyper-réactivité bronchique non allergénique (HBNA) représente la réduction excessive du calibre bronchique en réponse à divers stimulus non spécifiques; sans en être une caractéristique exclusive, l'HBNA est particulièrement importante dans l'asthme où elle joue un rôle essentiel dans la gravité des symptômes. Ses mécanismes pourraient associer, notamment, la réduction permanente du calibre bronchique par hyper-sécrétion, inflammation et œdème de la muqueuse à des anomalies de l'innervation bronchique et/ou de la réactivité des muscles lisses.

Jean-Luc Malo

Professeur agrégé de médecine à l'université de Montréal

Alain Lockhart

Professeur de physiologie à la faculté de médecine Cochin Port-Royal

\section{RÉFÉRENCES}

1. Tiffeneau R. Hypersensibilité cholinergohistaminique pulmonaire de l'asthmatique. Acto Allagol 1958 : 5 (suppl) 187-221.

2. Boushey HA, Holtzman MJ, Sheller JR, Nadel JA. Bronchial hyperreactivity. Am Rev Respir Dis 1980 ; 121 : 389-413.

3. Eiser N, Kerrebijn KF, Quanjer P. Guidelines for standardization of bronchial challenges with (non-specific) bronchoconstricting agents. Bull Eur Physiopathol Respir 1983 ; 19 : 495-514.

4. Woolcock AJ, Permutt S. Bronchial hyperresponsiveness. In : Macklem PT, Mead J, eds. Handbook of Physiology, Section 3. The respiratory system, Vol. 3. Mechanics of breathing, Part 2. Bethesda : American Physiological Society, 1986 : 727-36.

5. Freedman RJ. The functional geometry of the bronchi. The relationship between changes in external diameter and calibre, and a consideration of the passive role played by the mucosa in bronchoconstriction. Bull Eur Physiopathol Respir $1972 ; 8: 45-52$.

\section{ADRESSES}

J.-L. Malo : service de pneumologie, hôpital du Sacré-Cour et faculté de médecine, université de Montréal, 5400 ouest boulevard Gouin, Montréal, Canada H4J 1 C5.

A. Lockhart : laboratoire d'explorations fonctionnelles, hôpital Cochin, 27, rue du Faubourg-Saint-Jacques, 75014 Paris.
1 clair que l'hyperexcitabilité ou hyper-réactivité ou encore hypersensibilité des bronches est un composant essentiel de l'asthme, les fluctuations spontanées et provoquées de l'obstruction des voies aériennes étant d'autant plus grandes que l'hyper-excitabilité des bronches est plus accentuée. C'est à Tiffeneau que revient le mérite d'avoir, le premier, étudié de façon approfondie la sensibilité exagérée à l'histamine et à l'acétylcholine chez des asthmatiques [1]. De nombreux auteurs ont étudié depuis lors les mécanismes de ce phénomène et montré que d'autres agents pharmacologiques, chimiques et physiques (Tableau I) sont susceptibles de déclencher la réponse excessive caractéristique de l'hyper-réactivité bronchique non spécifique ou non allergénique (HBNA). Devant une telle variété d'agents, plusieurs questions viennent à l'esprit : d'abord quelle définition de l'HBNA doit-on retenir ? Ensuite quels sont les mécanismes de l'HBNA ? Et enfin, quelles sont les méthodes d'exploration de l'HBNA?

\section{Définition}

Les définitions récentes de l'hyper-réactivité bronchique non allergénique (HBNA) ont des points communs $[2,3,4]$ : en premier lieu, elles sont de type descriptif et opérationnel : l'HBNA est une réduction excessive de la perméabilité des bronches au courant de gaz en réponse à de nombreux stimulus ; ensuite, elles mettent en évidence que l'HBNA n'est pas spécifique de l'asthme ; enfin, elles ne donnent aucune information sur les mécanismes mêmes de l'HBNA, c'està-dire sur les modifications de structure et de fonction des bronches qui lui donnent naissance : les modifications fonctionnelles du poumon peuvent être causées par d'autres mécanismes que les changements de tension du muscle lisse des bronches, par exemple l'œdème de la muqueuse, la dilatation des vaisseaux de la muqueuse et les sécrétions. Le rôle de ces facteurs n'est pas clair [4].

\section{Mécanismes}

Les divers mécanismes susceptibles de donner naissance à l'HBNA sont représentés de façon schématique dans la figure 1 .

Réduction du calibre bronchique. La résistance à l'écoulement du gaz dans les bronches est fonction du calibre. Elle est inversement proportionnelle à la puissance quatrième du rayon dans les petites bronches où le débit est 
laminaire et à une puissance du rayon supérieure à quatre dans les grosses bronches où le débit est turbulent. Pour un même raccourcissement relatif du muscle lisse, par exemple $\mathrm{x} \%$ de sa longueur initiale, l'effet sur la résistance est d'autant plus grande que la muqueuse est anormalement épaisse, ou qu'il y a des sécrétions accumulées [5]. En pareil cas, la réponse excessive peut être indépendante d'une hyper-réactivité ou d'une hypersensibilité proprement dite, c'est-à-dire d'une contraction anormalement forte du muscle lisse à une dose donnée d'agoniste. Il en est de même quand les bronches sont précontractées avant l'épreuve.

Tout stimulus susceptible de faire contracter le muscle lisse (ou de causer l'épaississement de la muqueuse ou l'hyper-sécrétion) cause donc une augmentation d'autant plus importante de la résistance des voies aériennes que l'obstruction bronchique préexistante est plus sévère, par exemple dans l'asthme symptomatique [6]. Toutefois la corrélation trouvée en pratique entre le calibre initial des bronches, tel que l'on peut l'estimer en clinique, et l'intensité de la réponse bronchique à des stimulus non spécifiques est assez lâche aussi bien chez les sujets normaux [7] que chez des sujets atteints d'asthme [8] ou de bronchite chronique [9]. De plus à obstruction bronchique de base comparable, la réponse bronchique des sujets atteints de bronchite chronique à des stimulus non spécifiques n'atteint jamais l'extrême HBNA courante chez des asthmatiques symptomatiques. $\mathrm{La}$ réduction initiale du calibre bronchique n'explique donc pas totalement l'HBNA.

Anomalies du muscle lisse des bronches. Il y a accord sur l'existence d'une hypertrophie du muscle lisse des bronches d'asthmatiques. En revanche, l'existence d'anomalies intrinsèques des propriétés contractiles du muscle lisse est l'objet de controverses. Plusieurs études résumées par Hargreave et al. [10] ont montré qu'il n'y a pas de lien entre la réponse $m / s n^{\circ} 9$ vol. 3 , novembre 87

Tableau I

STIMULUS CAPABLES DE RÉVÉLER L'HYPER-EXCITABILITÉ BRONCHIQUE NON ALLERGÉNIQUE

1. Agents pharmacologiques

acétylcholine et ses dérivés (carbachol, méthacholine) histamine

prostaglandine $\left(\mathrm{PGF}_{2} \alpha, \mathrm{PGD}_{2}\right)$

dérivés de l'acide arachidonique $\left(\mathrm{LTC}_{4}, \mathrm{LTD}_{4}, \mathrm{LTE}_{4}\right)$

bradykinine

PAF-acéther

2. Agents physiques

exercice

hyperventilation d'air ambiant, froid ou sec

rire

3. Agents chimiques

acide citrique

eau distillée

solutions hypotoniques

solutions hypertoniques $(\mathrm{NaCl}, \mathrm{KCl})$

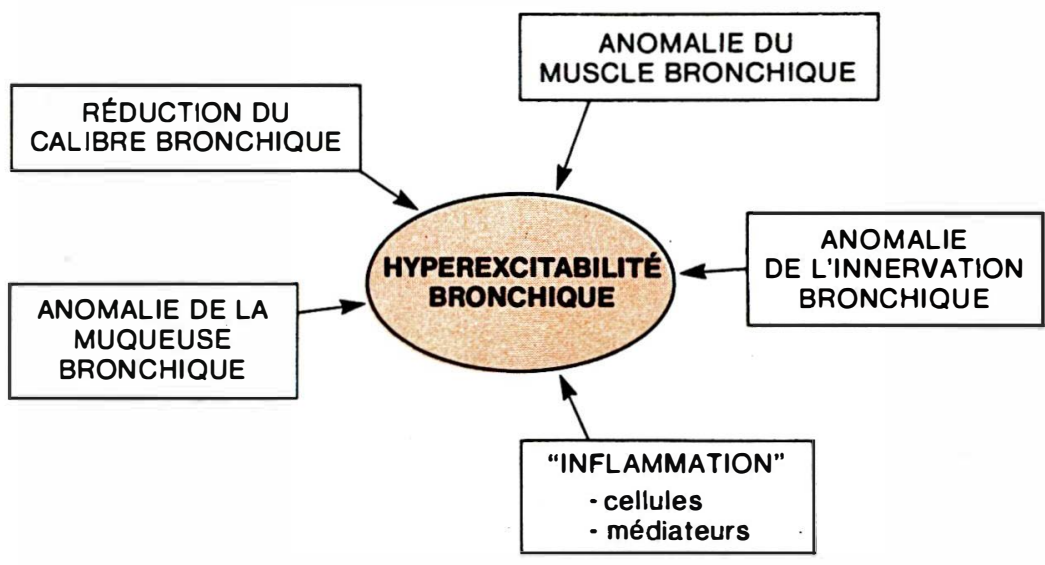

Figure 1. Mécanismes de l'hyper-excitabillté bronchique non spécifique. 
bronchique in vivo à des agents pharmacologiques comme l'histamine et la réponse contractile in vitro aux mêmes agonistes de bronches provenant des mêmes individus. Toutefois, une réserve est nécessaire. Dans toutes les études précitées, la réponse contractile mesurée est la force isométrique*. Or, divers travaux donnent à penser que la force isométrique in vitro peut être normale alors que d'autres éléments de la réponse contractile ne le sont pas. Macklem [11] a critiqué l'absence d'étude de la réponse isotonique $^{* *}$. Chez l'animal sensibilisé, la vitesse de raccourcissement et de décontraction in vitro de bronches à l'antigène est modifiée en l'absence d'hypertrophie musculaire [12, 13]. Il n'est pas impossible aussi que le muscle lisse des bronches acquière les propriétés d'un muscle "unitaire " chez des asthmatiques, c'est-à-dire un tonus myogène et une activité contractile spontanée [12]. Ces anomalies de la contraction du muscle lisse des bronches ont été mises en évidence dans différents " asthmes " expérimentaux. Mais elles sont discrètes et ont été décrites seulement sur des muscles provenant de grosses voies aériennes. Dans l'état actuel des connaissances elles ne peuvent pas expliquer les HBNA extrêmes de l'asthme humain.

Rôle de l'innervation des bronches. Le système nerveux autonome peut modifier de nombreuses façons le tonus ou la réponse réflexe des bronches. Toutefois la contribution d'anomalies fonctionnelles de l'innervation des bronches à l'HBNA est encore controversée $[14,15]$. La voie efférente vagale excitatrice entraîne la contraction du muscle lisse et la sécrétion des glandes bronchiques par l'intermédiaire de récepteurs post-jonctionnels muscariniques. L'existence de réflexes bronchoconstricteurs, dont le stimulus est

\footnotetext{
- Force mesurée au cours d'une contraction à longueur constante du muscle.

* Force mesurée au cours d'une contraction pendant laguelle le muscle se raccourcit en exerçant une force
}

l'excitation de terminaisons nerveuses de neurones afférents primaires nés dans la paroi des voies aériennes, est établie de façon indubitable dans différentes espèces animales. Des stimulus physiques (irritation mécanique), thermiques (passage d'air froid dans les voies aériennes), chimiques (histamine, par exemple) peuvent donner naissance à un tel réflexe bronchoconstricteur. Par ailleurs, certains médiateurs paracrines sont capables de moduler le fonctionnement de la voie efférente vagale. La transmission synaptique dans les ganglions parasympathiques de la trachée de furet est inhibée par la noradrénaline agissant sur des récepteurs $\alpha$ présynaptiques et la transmission cholinergique entre les terminaisons des neurones postganglionnaires et l'effecteur musculaire peut être modulée par des médiateurs la renforçant comme la sérotonine ou s'y opposant comme la prostaglandine $\mathrm{E}_{2}$.

Le système sympathique est inhibiteur par l'intermédiaire de l'adrénaline circulante qui pourrait jouer un rôle protecteur chez les sujets asthmatiques et par l'intermédiaire des neurones postganglionnaires destinés aux bronches. Chez l'homme, l'innervation directe du muscle lisse par des neurones post-ganglionnaires est clairsemée. En revanche, les vaisseaux systémiques bronchiques sont innervés et, comme nous l'avons vu précédemment, il existe des terminaisons noradrénergiques dans les ganglions parasympathiques où elles freinent la transmission synaptique. L'effet postjonctionnel de la noradrénaline est normalement inhibiteur de la contraction du muscle lisse des bronches du fait de la prépondérance des récepteurs $B$ adrénergiques, surtout $\beta_{2}$, sur les récepteurs $\alpha$ de type 1 et aussi de type 2 . Il est donc théoriquement possible que le système sympathique participe à l'HBNA soit du fait d'une activité réduite favorisant la transmission synaptique dans la voie cholinergique et diminuant l'effet inhibant direct des catécholamines sur le muscle lisse, soit par la réduction du nombre ou de l'efficacité des récepteurs $B$ adrénergiques post-jonctionnels, soit enfin par l'activation des récepteurs $\alpha$ adrénergiques. De tels événements peuvent être créés dans différentes espèces par des expériences dont la pertinence à l'HBNA de l'homme reste en grande partie à établir.

Il existe un second système efférent inhibiteur, responsable du relâchement des voies aériennes en réponse à la stimulation électrique [16]. Le médiateur est peut-être le VIP (vasointestinal peptide). La défaillance de ce systeme peut théoriquement jouer un rôle dans la production de l'HBNA. Enfin, des expériences de stimulation électrique en présence d'antagonistes cholinergiques ont mis en évidence l'existence d'un système excitateur non-adrénergique et non-cholinergique (système NANC). Il est constitué de neurones afférents primaires non myélinisés (fibres C) dont on trouve des terminaisons dans l'épithélium, le muscle lisse, les glandes et les vaisseaux. Ces neurones afférents primaires suivent la voie vagale et font relais dans le noyau du tractus solitaire. Les terminaisons contiennent des neurokinines (substance $P$, neurokinine A et neurokinine B). Cellesci ont de nombreux effets : vasodilatation, fuite de plasma par la paroi des veinules de la circulation systémique bronchique, contraction du muscle lisse. Des expériences ont prouvé que la stimulation de ces neurones afférents primaires peut entraîner la libération locale des neurokinines qu'ils contiennent, peut-être par un réflexe d'axone ${ }^{* * *}[15]$, et déclencher un ensemble de phénomènes de nature inflammatoire dans la paroi bronchique. Mais là aussi le rôle de ce système NANC excitateur dans l'HBNA, pour plausi-

\footnotetext{
** Après stimulation d'une terminaison réceptrice, le potentiel d'action suit dans le sens orthodromique le neurone afférent primaire vers les centres. Le potentiel d'action peut aussi parcourir dans le sens antidromique une collatérale et entraîner la libération de neuromédiateur par les terminaisons de celle-ci au contact d'un effecteur. C'est le réflexe d'axone.
} 
ble qu'il soit, n'est pas établi fermement.

Le rôle d'anomalies du système nerveux autonome des bronches dans la production de l'HBNA humain est difficile à établir. Il ne peut l'être que par des moyens pharmacologiques et dans la mesure où l'on dispose d'inhibiteurs spécifiques des neuromédiateurs possibles. Faute de tels inhibiteurs le rôle du système inhibiteur non adrénergique et du système NANC excitateur n'a pas été étudié chez l'homme. Des anomalies du système sympathique et du système cholinergique dans l'HBNA sont possibles. Le blocage de la transmission ganglionnaire par l'hexaméthonium inhalé réduit la réponse bronchique à l'histamine [15]. L'administration d'agents anticholinergiques réduit et, chez certains sujets, supprime la réponse bronchique à différents stimulus de l'HBNA tels que l'histamine, l'exercice ou l'hyperventilation $[15,17]$. Les agents $B$ bloquants non spécifiques (propranolol, par exemple) peuvent aggraver l'obstruction bronchique chez les sujets asthmatiques. L'ingestion de l'agoniste $\alpha_{2}$ adrénergique clonidine, qui réduit l'activité du système sympathique, augmente la réponse bronchique à $l^{\top}$ histamine beaucoup plus chez les sujets asthmatiques que chez les sujets normaux [18]. Mais il faut bien reconnaître qu'il s'agit là d'observations éparses et que le rôle du système nerveux autonome dans l'HBNA est loin d'être élucidé.

Rôle des lésions épithéliales et de l'inflammation. Il est de constatation courante que les infections virales aiguës des voies aériennes et, plus encore, l'exposition à des allergènes naturels ou professionnels auxquels le sujet est sensibilisé causent l'aggravation de l'asthme. Les lésions des bronches de sujets asthmatiques comportent la desquamation par endroits de l'épithélium, l'épaississement de la membrane basale dû au dépôt d'une protéine sécrétée par les polynucléaires éosinophiles, la protéine basique majeure (major basic protein, MBP), et des lésions inflammatoires associant la congestion, l'œdème et un inflitrat cellulaire à éosinophiles, polynucléaires neutrophiles, lymphocytes et mastocytes [10].

Diverses expériences ont confirmé l'hypothèse que de telles lésions pouvaient être responsables d'HBNA. Chez l'homme, l'inha- lation au laboratoire d'un allergène naturel ou professionnel auquel le sujet est sensibilisé peut causer trois variétés d'obstruction des voies aériennes selon leur évolution en fonction du temps (figure 2). Les réactions dites " semi-retardées " ou " doubles" (combinaison d'une réaction

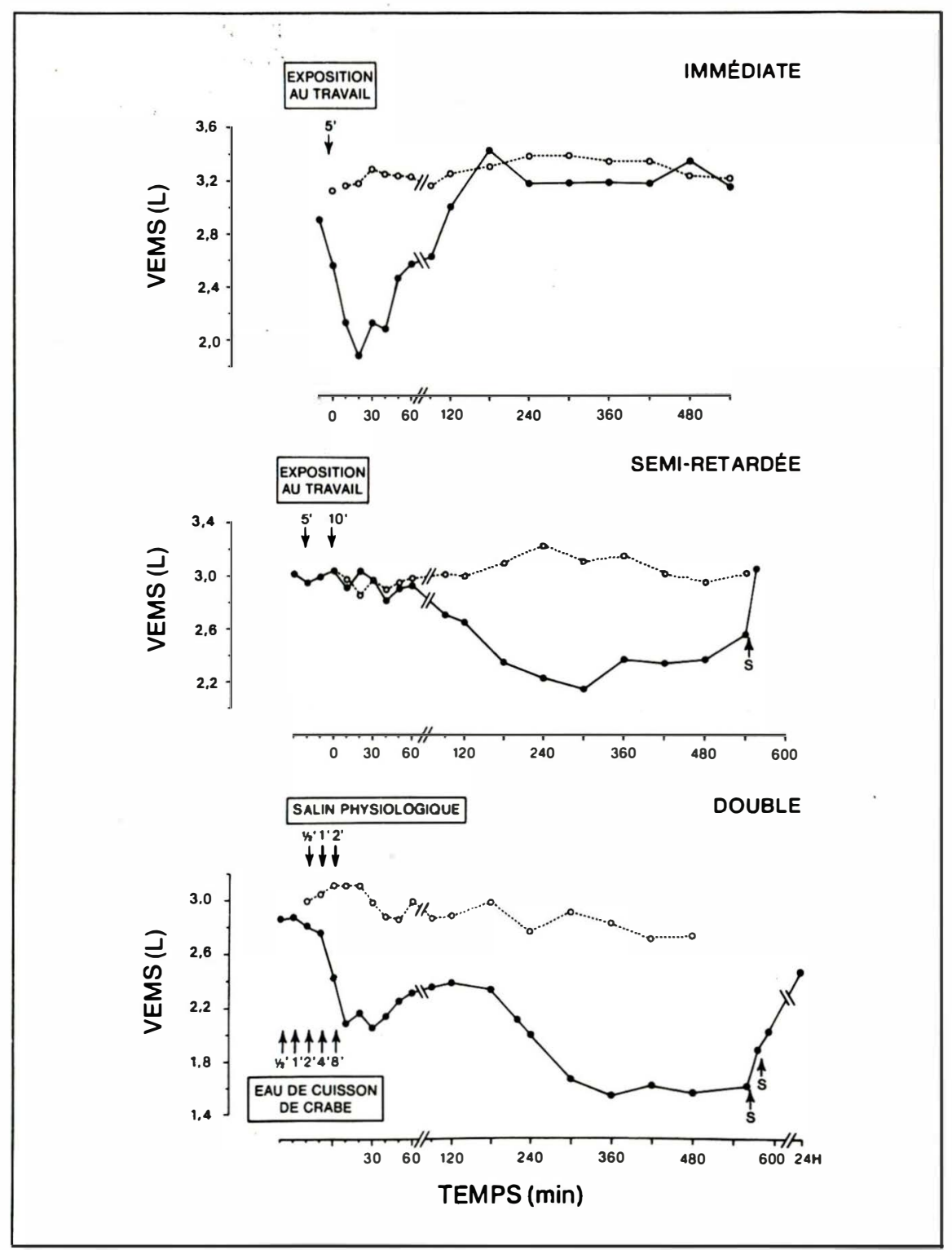

Figure 2. Changements du calibre bronchique mesuré par le volume expiratoire maximal par seconde (VEMS) (en ordonnée) après exposition au travail (cercles pleins, trait plein) et au laboratoire (pour témoin, cercles vides et trait pointillé) à un agent sensibilisant (eau de cuisson de crabe) pour des durées variables représentées par les flèches. Les trois types de réaction en fonction du temps sont illustrées. $S=$ administration aiguë de salbutamol (bronchodilatateur). 


\section{RÉFÉRENCES}

6. Orehek J, Gayrard P. Les tests de provocation bronchique non-spécifiques dans l'asthme. Bull Eur Physiopachol Respir 1976 ; 12 : 565-98.

7. Malo JL, Pineau L, Cartier A, Martin RR. Reference values of the provocative concentrations of methacholine that cause $8 \%$ and $20 \%$ changes in forced expiratory volume in one second in a normal population. Am Rev Respir Dis 1983 ; 128 : 8-11.

8. Cockcroft DW, Killian DN, Mellon JJA, Hargreave FE. Bronchial reactivity to inhaled histamine : a method and clinical survey. Clin Allergy $1977 ; 7: 235-43$

9. Bahous J, Cartier A, Quimet G, Pineau L, Malo JL. Non-allergic bronchial hyperexcitability in chronic bronchitis. Am Rev Respir Dis $1984: 129: 216-20$.

10. Hargreave FE, Dolovich J, O'Byrne PM, Ramsdale EH, Daniel EE. The origin of airway hyperresponsiveness. J Allergy Clin Immunuol $1986 ; 78: 825-32$.

11. Macklem PT. Bronchial hyporesponsiveness. Chest $1985 ; 87$ : 585.

12. Stephens NL. Postjunctional factors in airway smooth muscle hyperresponsiveness. In : Macklem PT, Mead J, eds. Hardbook of Physiology, Section 3. The respiratory system, Vol. 3. Mechanics of breathing, Part 2. Bethesda : American Physiological Society, 1986 : 719-2ó.

13. Souhrada JF, Dickey DC. Effect of antigen challenge on sensitized guinea pig trachea. Respir Physiol 1976 ; 27 : 241-51.

14. Nadel JA, Barnes PJ. Autonomic regulation of the airways. Ann Rev Med 1984; 35 : 451-67.

15. Barnes PJ. Neural control of human airways in health and disease. Am Rev Respir Dis $1986 ; 134: 289-314$

16. Richardson JB, Bouchard T. Demonstration of a nonadrenergic inhibitory nervous system in the trachea of the guina pig. J Allergy Clin Immunol 1975 ; 56 : 473-80.

17. Boulet LP, Latimer KM, Roberts RS, et al. The effect of atropine on allergen-induced increases in bronchial responsiveness to histamine. Am Rev Respir Dis 1984 ; 130 : 368-72.

18. Dinh Xuan T, Regnard J, Matran R, Mantrand P, Advenier C, Lockhart A. Effects of clonidine on bronchial responses to histamine and methacholine in normal and asthmatic subjects. Eur Respir $J$ (soumis pour publication).

19. Cartier A, Thomson NC, Frith PA, Roberts R, Hargreave FE. Allergen-induced increase in bronchial responsiveness to histamine : relationship to the late asthmatic response and change in airway caliber. $J$ Allergy immédiate et semi-retardée), par opposition avec les réactions immédiates isolées, sont associées à la libération et à l'activation de médiateurs chimiotactiques pour les polynucléaires [10] et causent des changements souvent prolongés de l'HBNA (figure 3) [19, 20, 21]. L'inhalation d'ozone par des sujets normaux cause une HBNA transitoire. Dans diverses espèces animales l'HBNA causée par l'ozone est associée à l'existence de lésions épithéliales et semble liée à l'apparition d'un infiltrat de la muqueuse à polynucléaires neutrophiles [10]. L'inhalation de PAF-acéther, un médiateur proinflammatoire doué d'ubiquité, entraîne chez des sujets normaux une HBNA durant plusieurs semaines [22]. Ces observations et ces expériences ont mis en évidence le fait fondamental que l'HBNA varie considérablement dans le temps chez un sujet donné, son maximum coïncidant avec différentes situations ayant en commun l'existence de lésions inflammatoires des bronches. L'un des buts du traitement de l'asthme est donc de réduire l'HBNA grâce à la lutte contre l'inflammation. Dans ce contexte, il -est paradoxal que des agents anti-inflammatoires puissants comme les stéroïdes parentéraux ou en inhalation aient relativement peu d'effet sur l'HBNA [10]. La difficulté d'interprétation de ces résultats pourrait provenir de ce que l'inflammation des bronches est un terme vague derrière lequel se cachent des réalités différentes. Dans l'état actuel des connaissances, diverses cellules et médiateurs impliqués dans ces réactions inflammatoires ont été identifiés : polynucléaires neutrophiles et éosinophiles, mastocytes, plaquettes [10].

Mais l'on ne sait pas comment l'état inflammatoire entraîne l'hypersensibilité des bronches à des agents chimiques et physiques aussi variés que ceux qui sont utilisés pour mettre en évidence l'HBNA. Tout au plus peut-on penser que la présence sur place de nombreuses cellules pouvant libérer des médiateurs capables d'attirer à leur tour de nouvelles cellules $\left(\mathrm{LTB}_{4}{ }^{*}, \mathrm{PAF}\right.$-acéther), de faire contracter le muscle lisse $\left(\mathrm{LTC}_{4}{ }^{*}, \mathrm{LTD}_{4}{ }^{*}, \mathrm{PGF}_{2} \alpha^{*}\right.$ ) et de causer l'extravasation de plasma et l'œdème (PAF-acéther, histamine) est responsable de la survenue de l'obstruction bronchique aiguë caractéristique de l'HBNA. De plus, certains de ces médiateurs, l'histamine par exemple, ainsi que les lésions épithéliales exposant les terminaisons nerveuses du système NANC excitateur pourraient être à l'origine de réflexes et de réflexes d'axone. Ces derniers en libérant localement des neurokinines pourraient aggraver l'œdème, la congestion et la contraction du muscle lisse. Enfin, in vitro, l'épithélium libère un médiateur paracrine s'opposant à l'effet contractant sur le muscle lisse de certains agonistes [23]. Mais l'on ne sait pas si un déficit de ce facteur relâchant épithélial joue un rôle dans l'HBNA.

\section{Mise en évidence de I'HBNA}

Comme il est indiqué dans le Tableau $I$, il y a de nombreuses façons de mettre en évidence l'HBNA. Toutes ces méthodes ont deux composants : le stimulus utilisé, particulier à chacune d'entre elles, et le mode d'appréciation de la réponse bronchique.

Parmi les stimulus pharmacologiques, l'acétylcholine et ses dérivés ainsi que l'histamine sont les plus anciennement utilisés. De nombreuses substances, pour la plupart considérées comme des candidats au rôle de médiateurs paracrines dans la maladie asthmatique, ont été ajoutées à cette liste. Quelle que soit la substance utilisée, il importe de suivre les recommandations publiées dont l'objet est de standardiser au mieux la quantité de substance inhalée, et les modalités de l'inhalation $[3,6,8,10]$. Le principe de base est d'administrer au sujet

\footnotetext{
- $L T B_{4}=$ leucotriène $B_{4} ; L T C_{4}=$ leucotriène $C_{4} ; L T D_{4}=$ leucotriène $D_{4} ; P G F_{2} \alpha=$ proslaglandine $F_{2} \alpha$.
} 


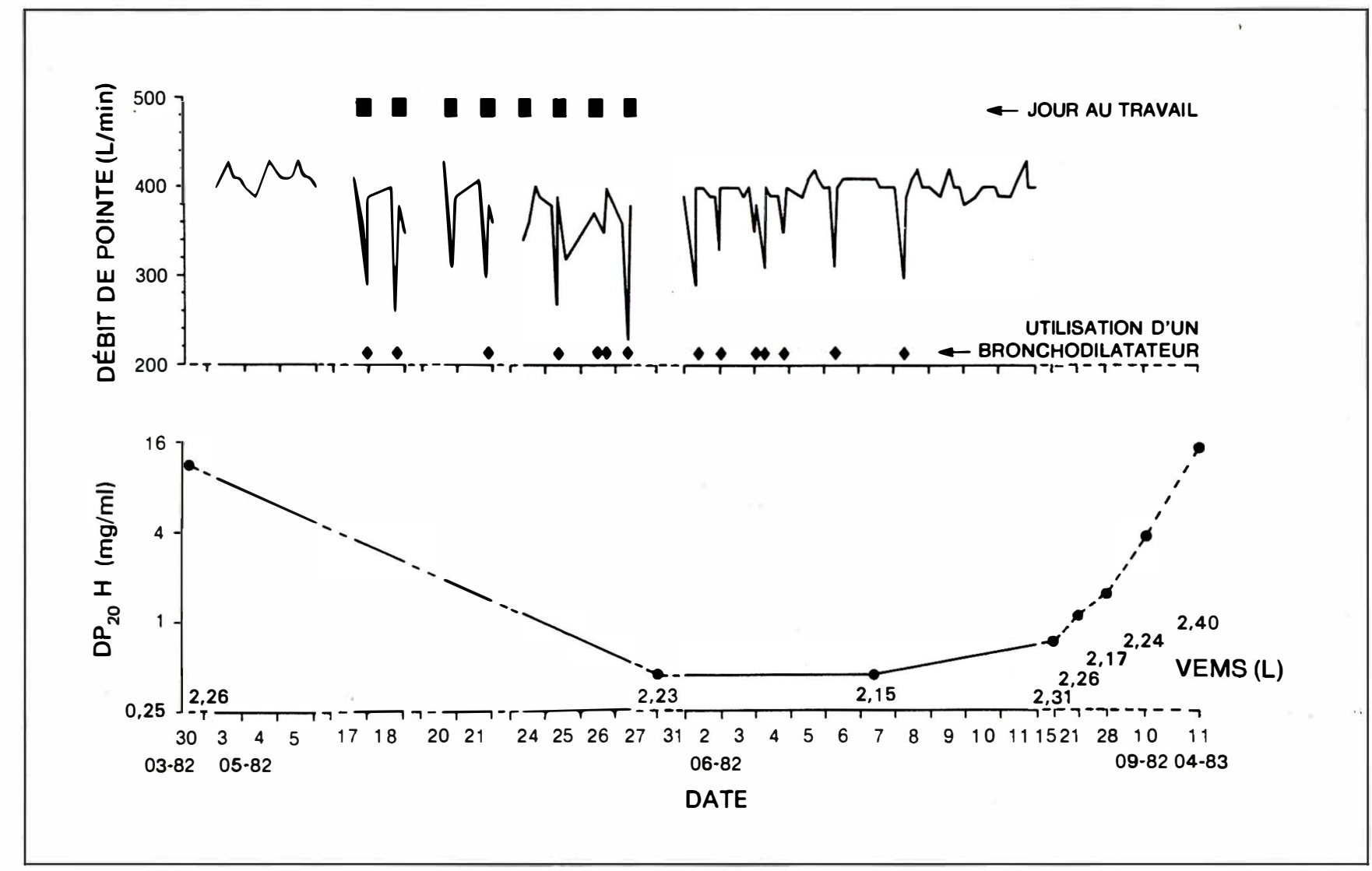

Figure 3. Changements du calibre bronchique mesuré par lo débit de pointe (partie supérieure de la courbe) et de I'HBNA mesurée par la concentration d'histamine inhalée en ventilation courante causant la baisse de $20 \%$ du VEMS $\left(D P_{20} H\right.$, partie inférieure de la courbe) après une période de huit jours au travail. Les losanges illustrent les moments d'utilisation d'un bronchodilatateur lors de symptômes d'asthme. Les valeurs de VEMS au moment de chacune des mesures de $D P_{20} \mathrm{H}$ sont illustrées et ne dépassent pas la valeur de base de plus de $10 \%$. Les changements de $D P_{20} \mathrm{H}$ induits par l'exposition au travail mettent environ un an pour se rétablir.

des doses successives du produit, chaque dose étant habituellement le double de la précédente et de mesurer la réponse bronchique après chaque dose. L'épreuve est arrêtée quand l'effet désiré, témoin du degré de l'obstruction bronchique provoquée, a été atteint. L'on obtient ainsi des courbes effet-dose par analogie avec ce qui est couramment fait en pharmacologie in vitro. Avec la plupart des substances utilisées la réponse des asthmatiques est obtenue avec des doses beaucoup plus faibles que chez le sujet normal, de 100 a 1000 ou 2000 fois plus faibles. Cependant la réponse d'asthmatiques asymptomatiques depuis des mois ou des années peut être normale. Celle des rhinites allergiques est intermédiaire $m / s n^{\circ} 9$ vol. 3, novembre 87 en moyenne entre celle des sujets normaux et celle des asthmatiques symptomatiques.

Au cours de ces dernières années, différents stimulus physiques ont été préconisés : au premier chef l'exercice physique [24]. L'obstruction bronchique consécutive à l'exercice tend à être d'autant plus sévère que la dissipation d'énergie dans les voies aériennes sous forme de perte d'eau et de chaleur est plus importante $[25,26]$ et ne se produit pas si la dissipation d'énergie est supprimée par l'inhalation d'air saturé en vapeur d'eau à la température du corps. L'hyperventilation isocapnique* a les mêmes effets que l'exercice pour peu que les propriétés physiques de l'air inhalé soient les mêmes. Pour une ventilation don- née, la dissipation d'énergie est d'autant plus importante que l'air inhalé est plus sec et plus froid. Ceci a donné naissance aux épreuves de provocation bronchique par inhalation d'air sec à température ambiante [27] ou d'air froid contenant donc très peu de vapeur d'eau [28]. L'hyperventilation a l'avantage sur l'exercice de permettre l'établissement de courbes effet-dose cumulatives [28], ce qui augmente la sensibilité de l'épreuve. Qu'il s'agisse d'exercice ou d'hyperventilation isocapnique, l'on admet que le stimulus est le refroidisse-

\footnotetext{
- Hyperventilation volontaire avec addition d'un débil de $\mathrm{CO}_{2}$ dans l'air inhalé, ajusté de façon à maintenir constante la pression partielle de $\mathrm{CO}_{2}$ dans le gaz expiré.
} 


\section{RÉFÉRENCES}

20. Hudson P, Cartier A, Pineau L, et al. Follow-up of occupational asthma caused by crab and various agents. J Allergy Clin Immunol 1985 ; 76 : 682-9.

21. Cartier A, Malo JL, Forest F, et al. Occupational asthma in snow crab-processing workers. J Allagy Clin Immunol 1984 ; 74 : 261-9.

22. Cuss FM, Dixon CMS, Barnes PJ. Effects of inhaled platelet activating factor on pulmonary and bronchial responsiveness in man. Lancet 1986 ; 2 : 189-92.

23. Frossard N, Muller F. Epithelial modulation of tracheal smooth muscle responses to antigenic stimulation. J Appl Physiol 1986 ; 65 : 1449-56.

24. Lockhart A, Regnard J, Dessanges JF, Florentin D, Lurie A. Exercise and hyperventilation-induced asthma. Bull Eur Physiopathol Respir 1985 ; 21 : 399-409.

25. Deal EC, McFadden ER, Ingram RH, Strauss RH, Jaeger JJ. Role of respiratory heat exchange in production of exerciseinduced asthma. $J$ Appl Physiol 1979 ; 46 : 467-75.

26. Anderson SD. Is there a unifying hypothesis for exercise-induced asthma ? J Allergy Clin Immnuol $1984 ; 73$ : 660-5.

27. Denjean A, Matran R, Mathieu M, Cerrina J, Duroux $P$, Lockhart A. Bronchial response to hyperventilation of dry air at room temperature in normals and asthmatics. Bull Eur Physiopalhol Respir 1983; 19 : 477-82.

28. Malo JL, Cartier A, L'Archevêque J, Ghezzo H, Martin RR. Cold air inhalation has a cumulative bronchospastic effect when inhaled in consecutive doses for progressively increasing degrees of ventilation. Am Rev Respir $D$ is 1966 ; 134 : 990-3.

29. O'Byrne PM, Jones GL. The effect of indomethacin on exercise-induced bronchoconstriction and refractoriness after exercise. $A m$ Rev Respir Dis 1986 ; 134 : 69-72.

30. Weiler-Ravell D, Godfrey S. Do exercise - and antigen - induced asthma utilize the same pathways. J Allergy Clin Immunol 1981 ; $67: 391-7$.

31. Anderson SD, Schoeffel RE, Finney $M$. Evaluation of ultrasonically nebulised solutions for provocation testing in patients with asthma. Thorax 1983 ; 38 : 284-91.

32. Allegra L, Bianco S. Non-specific bronchoreactivity obtained with ultrasonic aerosol of distilled water. Eur J Respir Dis 1980 ; 106 : 41-9.

33. Bascom R, Bleecker ER. Bronchoconstriction induced by distilled water. Am Rev Respir ment de la muqueuse des voies aériennes ou l'hyperosmolarité du liquide tapissant les voies aériennes $[25,26]$. Mais les mécanismes intimes mis en jeu ne sont pas connus et il y a plusieurs raisons de penser qu'ils ne sont pas les mêmes dans tous les cas. Tout d'abord, dans un cas sur deux environ et dans les deux heures suivant une première crise d'asthme post-exercice et posthyperventilation, il existe une phase réfractaire, sans doute liée à la production de prostaglandines vasodilatatrices [29] ; par phase réfractaire, on entend qu'un deuxième stimulus identique au premier ne cause pas de réponse bronchique ou une réponse moindre que la première. Ensuite, au cours de cette phase réfractaire, la stimulation bronchique par un allergène auquel le sujet est sensible déclenche une réponse dans environ la moitié des cas seulement [30]. Puis, l'existence d'une réponse tardive, analogue à celle provoquée par certaines stimulations des bronches par des allergènes, existe dans un petit nombre seulement d'asthmes postexercice [10]. Enfin, l'asthme post-exercice ou post-hyperventilation n'est totalement prévenu par l'administration d'anticholinergiques que dans environ la moitié des cas.

L'HBNA peut être révélée par d'autres stimulus physiques, l'inhalation de solutions hyperosmolaires par exemple [31], ce qui est l'un des arguments en faveur du rôle de la dessication des voies aériennes comme facteur déclenchant de l'asthme post-exercice ou post-hyperventilation. La portée de cet argument est toutefois réduite par le fait que l'inhalation d'eau distillée pulvérisée est aussi capable de déclencher une réponse bronchique chez des asthmatiques, pour des quantités d'eau inhalée, dépourvue d'un tel effet chez les sujets normaux $[31,32]$. Chez des asthmatiques ayant une hyperréactivité à la méthacholine, les réponses précoces à l'exercice et à l'eau distillée sont liées entre elles mais inconstantes. Il est possible que les répondeurs et les non-répondeurs à l'eau distillée forment deux sous-groupes distincts sans que l'on sache le mécanisme de cette différence [33]. Il y a deux méthodes pour apprécier la réponse bronchique. La première est de mesurer la résistance à l'écoulement du gaz dans les voies aériennes; comme celleci dépend du volume du poumon, celui-ci doit être mesuré conjointement, ce qui impose l'emploi d'un pléthysmographe* corporel. La seconde est de mesurer les débits expiratoires maximaux. Ceci peut être fait à partir d'une expiration forcée consécutive à une inspiration maximale ; c'est ainsi qu'est mesuré le classique VEMS (volume expiré pendant la première seconde d'une expiration maximale). Mais l'inspiration maximale initiale a un effet bronchomoteur qui peut modifier la réponse [6]. Cet inconvénient est contrebalancé par la meilleure reproductibilité du VEMS que de la résistance des voies aériennes et est éliminé si l'expiration forcée démarre à partir d'un gonflement partiel du poumon dans la manœuvre permettant d'obtenir des courbes débit-volume partielles. Celles-ci permettent seulement de connaître les débits expiratoires maximaux à des fractions connues de la capacité pulmonaire totale.

Il y a aussi plusieurs façons d'analyser les résultats. L'une des plus courantes est de déterminer l'intensité d'un stimulus (dose d'agoniste, ventilation pulmonaire) entraînant la chute, considérée comme significative, des débits expiratoires maximaux. On exprime habituellement la réponse sous la forme de la dose d'agoniste ou de la ventilation causant un effet prédéterminé, par exemple chute de $20 \%$ du VEMS

\footnotetext{
- On appelle pléthysmographe tout appareil permetlant la mesure des variations de volume d'un organe ou d'une partie de l'or ganisme. La pléthysmographie corporelle est un cas particulier : le sujet est enfermé dans un caisson rigide où l'on mesure pendant qu'il respire à l'extérieur, les variations de volume (ou de pression) de l'air contenu dans l'enceinte. A partir des grandeurs mesurées, on peut calculer le volume du compartiment gazeux intrathoracique el ses varialions pendant la ventilation.
} 
$\left(\mathrm{DP}_{20}\right.$ ou $\dot{\mathrm{V}}_{20}$, respectivement) ou l'accroissement de la résistance des voies aériennes de $40 \%$ par exemple. Il existe d'autres façons d'analyser les courbes dose-effet. On peut calculer par exemple l'intensité du stimulus qui entraîne un effet significatif (dose seuil) et la pente de la courbe dose-effet au-delà du seuil [6]. Bien qu'un indice comme $\mathrm{DP}_{20}$ ait fait l'objet de la meilleure évaluation clinique et soit le plus utilisé, les avantages et inconvénients respectifs des différentes façons d'analyser les résultats font l'objet de controverse [3]. Quelle que soit la méthode utilisée, ce sont les conséquences de la réponse bronchique sur l'écoulement du gaz dans les voies aériennes qui sont mesurables et mesurées et non pas la réponse du muscle lisse bronchique elle-même. Ceci justifie l'emploi des définitions purement descriptives de l'HBNA citées au début de cet article et les incertitudes persistantes sur les composants mêmes de cette réponse.

\section{Indications des épreuves de provocation bronchique}

Il y a plusieurs indications à la mesure de l'HBNA. Schématiquement, la mise en évidence de l'HBNA peut servir au diagnostic de l'asthme, améliorer la connaissance de celui-ci, suivre les effets du traitement et aider, ce faisant, à améliorer les comportements thérapeutiques. Il est des cas où le diagnostic clinique d'asthme est incertain soit qu'il y ait des difficultés de communication entre le malade et le médecin, soit que le sujet se plaigne de symptômes qui ne sont pas caractéristiques comme une dyspnée ou une toux rebelle, soit enfin qu'il n'y ait pas d'obstruction bronchique de base ou que l'on n'obtienne pas, par les examens spirométriques usuels ou la mesure pluriquotidienne du débit expiratoire de pointe avec un appareil portatif, la preuve de l'obstruction bronchique, rapidement changeante, constitutive de l'asthme. La mise en évidence d'une HBNA est alors un argu- ment important en faveur de ce diagnostic.

L'HBNA est un des composants de l'asthme à côté de la capacité qu'ont certaines variétés de cellules, notamment les mastocytes, à sécréter des médiateurs en réponse à des stimulus, allergènes par exemple [10]. Par conséquent l'étude des stimulus capables de révéler l'HBNA et celle des modalités de la réponse bronchique à ces stimulus peut contribuer à mieux comprendre la maladie asthmatique ; c'est ainsi notamment qu'ont été mis en évidence le rôle aggravant des lésions épithéliales et de l'inflammation.

Suivre l'évolution de l'HBNA sous traitement paraît utile. Plus l'HBNA est grande et plus les variations spontanées, notamment circadiennes, de l'obstruction bronchique sont importantes, en particulier l'aggravation de la seconde partie de la nuit qui peut être fatale. Réduire l'HBNA par le traitement est alors souhaitable. L'on commence à réaliser que l'on dispose de deux catégories schématiques de médicaments : les bronchodilatateurs comme les $\beta_{2}$-agonistes inhalés, dont l'effet est surtout symptomatique mais qui ne réduiraient pas I'HBNA, et les médicaments antiinflammatoires comme les corticoïdes, administrés de plus en plus par inhalation, ou les médicaments anti-allergiques comme les cromones. Ces deux dernières catégories de médicaments sont sans effet immédiat sur les symptômes mais semblent bien agir en réduisant progressivement les lésions inflammatoires des bronches, améliorant ainsi progressivement les symptômes de la maladie. Enfin l'évaluation répétée de l'HBNA est utile à la recherche sur l'asthme professionnel et à l'expertise médico-légale le concernant. Comme nous l'avons vu, le contact avec' un allergène professionnel sensibilisant par opposition avec le contact avec un agent irritant entraîne le plus souvent l'augmentation durable de l'HBNA (figure 3) persistant au-delà de la cessation de l'exposition

\section{Summary}

Although it is not specific for asthma, non-specific or nonallergenic bronchial hyperresponsiveness is the hallmark of this condition together with the rapid changes in airway calibre occurring either spontaneously or as a result of therapy. The present article is a state of the art on bronchial hyperresponsiveness. Current definitions of bronchial hyperresponsiveness state that $: 1$ ) it is characterized by a reduction in airway calibre after the application of a stimulus ; 2) it is not specific for asthma ; 3) its mechanisms are unknown. The possible mechanisms of bronchial hyperresponsiveness are also reviewed. These include : 1) reduction in airway calibre and/or increased thickness of the airways wall ; 2) abnormalities of bronchial smooth muscle ; 3) abnormalities of bronchial innervation ; 4) epithelial damage and inflammation. The ongoing research on and clinical means for the assessment of bronchial hyperresponsiveness through the use of pharmacological, physical and chemical agents are described. Finally, the indications for measuring bronchial hyperresponsiveness are discussed.

\section{TIRÉS À PART}

J.-L. Malo : service de pneumologie, hôpital du Sacré-Cœur et faculté de médecine, université de Montréal, 5400 ouest boulevard Gouin, Montréal, Canada H4J 1C5. 